\title{
3D Models of Exoplanet Atmospheres
}

\author{
Ian Dobbs-Dixon \\ Department of Astronomy, University of Washington \\ Box 351580, Seattle, WA 98195 \\ Sagan Postdoctoral Fellow \\ email: ianmdd@gmail.com
}

\begin{abstract}
Dynamical models of strongly irradiated gas-giant atmospheres exhibit a range of behavior, the nature of which depends on both the adopted parameters and the adopted numerical model. Discerning the correct choice of physical parameters and modeling philosophy can be difficult. Here, I present a series of wavelength-dependent transmission spectra for the giant planet HD209458b based on 3D radiative hydrodynamical models for a range of kinematic viscosities. While flow patterns and temperature distributions can vary significantly, disk-averaged phase curves mask much of this information. Transmission spectra, on the other hand, probe the day-night transition where advective contributions dominate and differences are often most pronounced. Transmission spectra illustrate noticeable changes, especially when comparing the differences between transmission spectra of eastern and western hemispheres, as might be seen during ingress and egress.
\end{abstract}

Keywords. hydrodynamics, radiative transfer, techniques: spectroscopic, eclipses

\section{Introduction}

Within the last decade, there has been an explosion in both transiting extrasolar planets and new observational techniques aimed at determining interior and atmospheric composition, dayside temperature, and efficiency of energy redistribution. In addition to primary transit measurements (Charbonneau et al. 2000), these tools include secondary eclipse (Deming et al. 2005), differential spectroscopy (Charbonneau et al. 2002), phase-curve monitoring (Harrington et al. 2006, Cowan et al. 2007), Doppler absorption spectroscopy (Snellen et al. 2010), and transmission spectra (Brown et al. 2002). These observations have motivated many groups to study the atmospheric dynamics of these planets and its role in both redistributing incident stellar energy throughout the atmosphere and (potentially) in influencing the overall evolution of the interior. Multiple groups are working on coupled radiation-hydrodynamical solutions with multiple assumptions and approaches. See Dobbs-Dixon et al. (2010) for a list of approaches to both the dynamical and radiative portions of the model.

One common feature of many simulations of the short period planets HD209458b and HD189733b are strong circumplanetary, eastward equatorial jets. Observations of HD189733b (Knutson et al. 2007, 2009; Agol et al. 2010) appear to have convincingly demonstrated the existence of equatorial super-rotation, showing that the hottest point in the phase curve appears slightly before secondary eclipse, as first predicted by Showman $\&$ Guillot (2002). However, as we are able to characterize more planets, we are finding a wide variety of behaviors. For example, observations of $\nu$-Andromeda B (Crossfield et al. 2010) indicate a much larger phase shift of $\approx 89^{\circ}$, completely out of the range of current model predictions. In addition, models with varying viscosity (Dobbs-Dixon et al. 2010), perhaps due to magnetic drag, sub-grid turbulence, or shocks, indicate that the circumplanetary jet may not be as universal as once thought. 
Unfortunately, much of the detailed spatial and temporal structure that is seen in numerical simulations is hidden in the necessarily hemispherically averaged phase curves (Cowan et al. 2008). Temperature differences across jets, latitudinal dependence, vortices, and other interesting sub-hemisphere scale phenomena largely disappear. However, one technique that may prove quite useful in this respect is transmission spectroscopy. Taken as the planet transits its host star, transit spectroscopy measures the absorption of stellar light by the upper limbs of the planetary atmosphere yielding a wavelength-dependent radius for the planet (Seager et al. 2000). Given the viewing geometry of the star, planet, and observer, such a measurement probes the meridians delineating the day and night hemispheres (the terminators). The variation in opacity with wavelength can cause the planet to vary in absorption radius by $\approx 5 h$, where $h$ is the atmospheric scale height, leading to depth variations on the order of $10 R_{p} h / R_{*}^{2} \approx 0.1 \%$ for $5 h \approx 3500 \mathrm{~km}, R_{p} \approx R_{J}$, and $R_{*} \approx R_{\odot}$.

Dynamics play a crucial role in shaping the temperatures across the terminators both at the surface and at depth. It is here that one expects the largest deviations from radiative equilibrium. As high velocity jets advect energy across the terminator to the night-side, the flow will cool radiatively, thus one would expect the largest night-side temperatures to be closest to the terminator. Indeed, simulations show similar behavior, but the exact temperature distribution depends sensitively on the details of the flow structure and radiative and advective efficiencies. Transmission spectra taken both at mid-transit and during ingress and egress have great potential to help distinguish between models and adopted parameters. Several models presented in Dobbs-Dixon et al. (2010) show pronounced variability with the largest amplitudes at the terminator region. Targeting the terminator regions with multiple transmission spectral measurements may reveal spectral changes due to such dynamically driven weather.

Here, I utilize the 3D pressure-temperature profiles calculated using the 3D NavierStokes equations and a multi-channel flux-limited diffusion. Models differ from DobbsDixon et al. (2010) in several important respects; I now allow for advective flow over the polar regions and the stellar energy deposition is now wavelength-dependent. Section 2 briefly describes the methods for calculating transmission spectra from multi-dimensional models. In Section 3, I present several diagnostics utilizing these transmission spectra that may be detectable with the next generation of instruments. I conclude in Section 4 with a discussion of the relevance of this work for compact, mass-transferring binaries.

\section{Transit Spectra Calculations}

To calculate the pressure and temperature throughout the atmosphere of HD209458b, we utilize a fully non-linear, coupled radiative hydrodynamical code. We solve the fully compressible Navier-Stokes equations throughout the 3D atmosphere together with coupled thermal and radiation energy equations. Direct stellar heating of the planet is taken into account through a spatial and wavelength-dependent source term. Local 3D radiative transfer and cooling are included through flux-limited diffusion. Wavelengthdependent opacities are taken from the atomic and molecular opacity calculations of Sharp et al. (2007). For mode detailed information on the dynamical model, see DobbsDixon et al. $(2010,2011)$.

To determine the transmission spectra of HD209458b models, I calculate the wavelength-dependent absorption of stellar light traversing through the limb of the planet. This allows the determination of the effective radius of the planet and a fractional reduction of stellar flux $F_{\star}$. Neglecting limb-darkening of the star, this can be 
expressed as $\left(\frac{F_{\text {intransit }}}{F_{\star}}\right)_{\lambda}=\frac{\int\left(1-e^{\tau(b, \phi, \lambda)}\right) b d b d \phi}{\pi R_{\star}^{2}}$, where $\tau(b, \alpha, \lambda)$ is the total optical depth along a given chord with impact parameter $\mathrm{b}$ and polar angle $\alpha$, defined on the observed planetary disk during transit. The density and temperature needed to calculate $\tau$ at each location are interpolated from the values in the $3 \mathrm{D}$ models.

Several other groups have also explored the differences between transmission spectra calculated with 1D or 3D models. Fortney et al. (2010) and Burrows et al. (2010) perform similar calculations utilizing the 3D GCM models of Showman et al. (2009) and Rauscher et al. (2010), respectively. As mentioned above, the dynamical calculations differ amongst these models and ours, but the method for calculating the resulting spectra from the results is largely equivalent.

\section{Calculated Transmission Spectra}

In this Section, I present transmission spectra from the 3D models exploring the different flow structures among models with varying viscosity. While several features may influence the transmission spectra at precisions already observed, others may await the next generation of instruments. I highlight two methods for extracting this signal from actual data, exploiting the differences between transmission spectra taken during transit ingress (probing the eastern terminator) and transit egress (probing the western hemisphere).

Transmission spectra calculated from 3D models with varying viscosities are shown in Figure 1. The variations due to varying flow structures (see Figures 2 and 3 of DobbsDixon et al. (2010) for an illustration of the differences) can be quite dramatic, including a transition from sub-sonic to supersonic wind speeds as the viscosity is lowered. Simulations S1, S2, S3, and S4 have viscosities of $10^{12}, 10^{10}, 10^{8}$, and $10^{4}$, respectively.

Short period, synchronously rotating, irradiated planets are not spherical due to a number of reasons including rotation, day-night temperature gradients, equator-pole temperature gradients, and the tidal potential of the host star. For a slow rotating planet such as HD209458b, the temperature gradients across the planet (yielding different scale-heights) are the largest factor in changing the planet's shape.

The first method we discuss is wavelength-dependent transit timing. As the western terminator has a smaller scale height and this part of the planet transits first, the ingress will be slightly delayed, while the eastern terminator is more extended causing the end of ingress to be delayed. Likewise, the egress will be delayed as well, so the overall shift
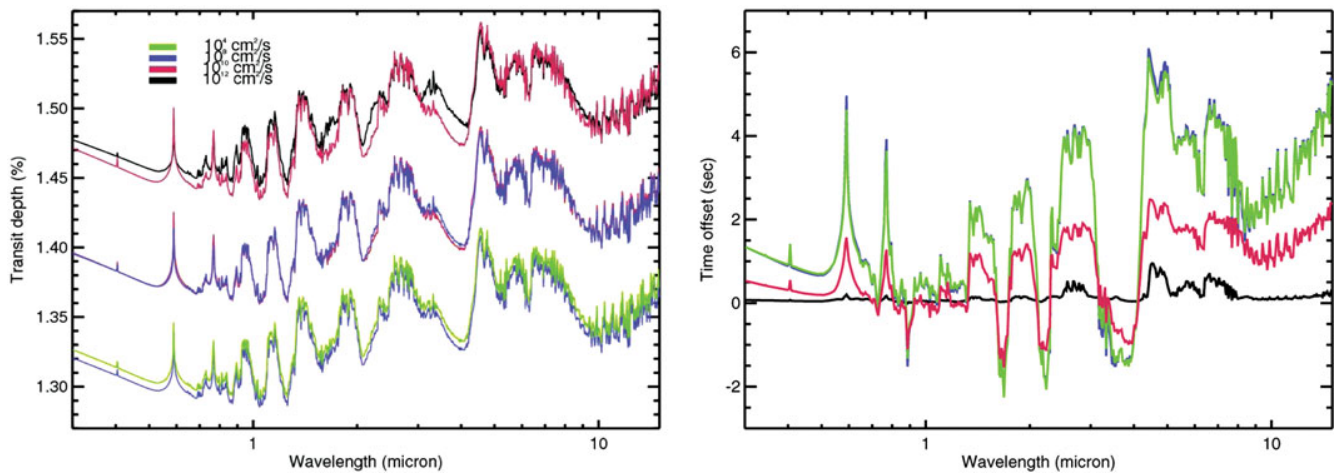

Figure 1. Difference in mid-transit transmission spectra among models with varying viscosity (left). Effective time offset versus wavelength (right). Black, red, green, and blue lines are from $\mathrm{S} 1, \mathrm{~S} 2, \mathrm{~S} 3$, and $\mathrm{S} 4$ simulations respectively. 
will be a delay of the transit relative to the center of mass of the planet. At wavelengths with larger opacity, this asymmetry is stronger so the transit time delay is larger, while at wavelengths with smaller opacity, it is weaker; consequently, the central time of transit will appear to vary with wavelength if one fits the transit with a symmetric planet model. Figure 1 shows the effective transit time offset versus wavelength computed for a model with no limb-darkening for the star and by fitting the transit at each wavelength with a circular planet model (Mandel et al. 2002). At some wavelengths, the transit time is offset earlier, which is caused by a change in the opacity at certain wavelengths due to chemistry differences between the terminators. As the viscosity grows, the two hemispheres have smaller temperature differences, and hence the transit shape is more symmetric, causing a smaller time offset.

Another model-independent diagnostic for the planet asymmetry is the color-dependent transit shape. Figure 2 shows the difference in the shape of transit for two wave bands: 1.55-2.42 and 1.70-3.1 micron, corresponding to the JWST NIRCam F162M and F277W filters, respectively. To compute the shape difference, the depths of the transit in each band were divided by the maximum depth, and then subtracted from one another: $C=\left(D_{1} / D_{1, \max }-D_{2} / D_{2, \max }\right)$ where $D_{1,2}$ is the depth of transit (in dimensionless units: i.e. the out-of-transit flux minus the in-transit flux divided by the out-of-transit flux), and the max subscript indicates the maximum depth of transit. The asymmetry in these light curves demonstrates that at different wavelengths the planet absorbs with a different asymmetric cross section. These filters have the advantage that they can be observed simultaneously with JWST using the dichroic and the first band has a low water opacity, while the second has a higher water opacity for this particular model.

\subsection{Comparison to observations}

As a check on the total depth of transit, we have compared the transit depth to observations by Knutson et al. (2008) and Beaulieu et al. (2010). These observations cover a wavelength from 0.3 to 10 microns, and thus provide an important validation test of these numerical models. We exclude the bands with strong sodium and potassium absorption as there is evidence that potassium is depleted (which is not accounted for in our opacity tables), while the sodium absorption occurs quite high in the atmosphere, and
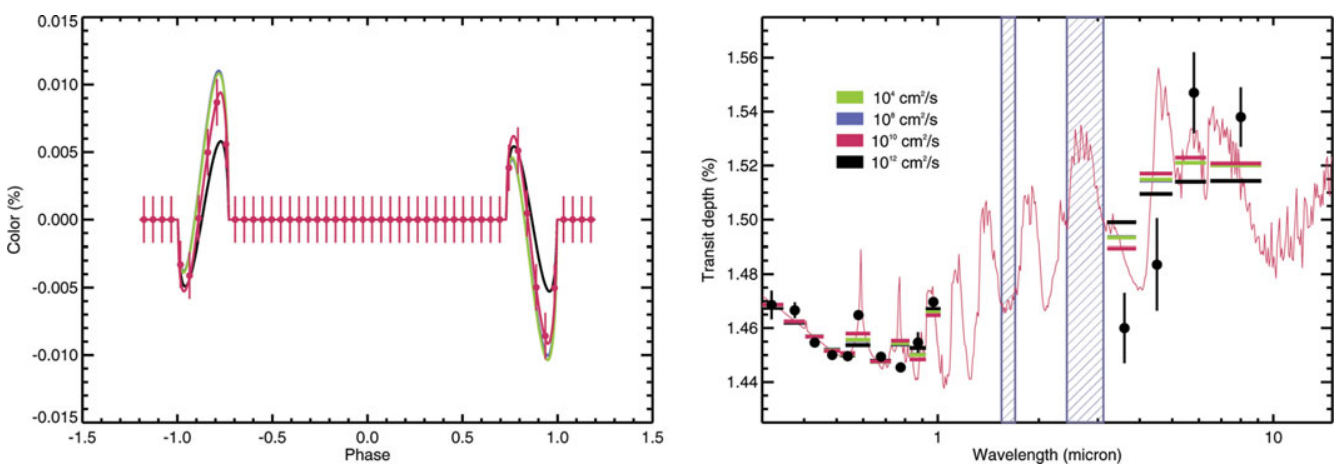

Figure 2. Color of transit for the F162M minus F277W JWST NIRCam filters (left). Transit depth versus wavelength (right). The black dots with error bars are the data from Knutson et al. (2007) and Beaulieu et al. (2010). The thick horizontal bars are the four models averaged over the measured wave bands. The light red curve is the $10^{10} \mathrm{~cm}^{2} \mathrm{~s}^{-1}$ model binned by a factor of 10 in wavelength; this is over-plotted as it had the best fit of all four models (although not statistically significant) 
thus cannot be handled in our models. We have averaged the models over the observed bands, and have varied the value of the inner radius of the simulation zone to obtain the best agreement with the observed transit depths. This is not as accurate as carrying out additional simulations in which the model region is varied; however, this would be much more computationally expensive. Furthermore, the assumption of constant $\mathbf{g}$ in the simulations of Dobbs-Dixon et al. (2010) and other groups allows for such a shift as only the curvature terms are affected.

There are twelve wave bands that we use, and with one free parameter, gives eleven degrees of freedom for each model. Figure 2 shows the comparison of the models to the data. We find best-fit chi-squares of 23.9, 24.1, 23.6, and 28.3 for the models with viscosities of $10^{4}, 10^{8}, 10^{10}$ and $10^{12} \mathrm{~cm}^{2} \mathrm{~s}^{-1}$, respectively. Qualitatively, the overall agreement of the models with the data is quite good: (1) the transit depth is weakly dependent on the viscosity; (2) there are no discrepancies between the data and model greater than $2-\sigma ;(3)$ the observed transit spectrum with wavelength shows the expected features due to water and Rayleigh scattering. In general, the success of this model is comparable to transmission spectra calculated from other models which utilize GCM simulations (Fortney et al. 2010, Burrows et al. 2010).

However, in detail there are significant discrepancies; in particular, the observed IRAC transit depths appear to vary more strongly with wavelength than the model predicts. This is reflected in the larger chi-square of the fits, of which two-thirds is due to the infrared discrepancies. The fit to the infrared data obtained by Beaulieu et al. (2010) has an extremely good chi-square; however, their model was one-dimensional, and allowed the abundances and temperature-pressure profile to float, so it is not surprising that they obtain a good fit with so many degrees of freedom. Another possibility is that systematic errors still exist in the IRAC data reduction. For example, for the transiting planet HD 189733, different groups have obtained markedly different transit depths at infrared wavelengths using the same IRAC data sets; consequently, there may be some remaining systematic error present in the data (Desert et al. 2009). The final possibility is that there is still physics that are not included in our models which are causing the discrepancies; for example, varied chemical abundances, non-equilibrium chemistry, and magnetic drag (yielding non-isotropic viscosities) have not been included in these models.

\section{Discussion}

In these proceedings, I have presented theoretical transmission spectra of HD209458b calculated from full 3D radiative-hydrodynamical models. In general, there is good agreement with observational spectra though some discrepancies remain. I have highlighted 2 new observational techniques for deducing the strength of a possible circumplanetary equatorial jet. These include wavelength-dependent transit timing and color-dependent transit shapes. Both methods may be applicable to the next generation of instruments.

Simulations of highly irradiated atmospheres of exoplanets are relevant for the binary community at large. In particular, the processes occurring on the surfaces of lower-mass, shorter-period binaries will likely resemble the results presented here. Tidal locking in these systems is expected to occur rapidly, resulting in permanent day-night temperature differences. The pressure gradient associated with this will drive strong winds altering both observables (as discussed here) and potentially mass-transfer processes. Although the subject has been studied to some extent, much work remains before a coherent picture of the atmosphere dynamics in binaries emerges. 


\section{References}

Agol, E., Cowan, N. B., et al., 2010, ApJ, 721, 1861

Beaulieu, J. P., Kipping, D. M., Batista, V., et al., 2010, MNRAS, 409, 963

Brown, T. M., Libbrecht, K. G., \& Charbonneau, D. 2002, PASP, 114, 826

Burkert, A., Lin, D. N. C., et al., 2005 ApJ, 618, 512

Burrows, A., Rauscher, E., Spiegel, D. S., \& Menou, K. 2010, ApJ, 719, 341

Charbonneau, D., Brown, T. M., Latham, D. W., \& Mayor, M. 2000, ApJ, 529, L45

Charbonneau, D., Brown, T. M., Noyes, R. W., \& Gilliland, R. L. 2002, ApJ, 568, 377

Cho, J. Y. K., Menou, K., Hansen, B. M. S., \& Seager, S. 2003, ApJ, 587, L117

Cho, J. Y. K., Menou, K., Hansen, B. M. S., \& Seager, S. 2008, ApJ, 675, 817

Cooper, C. S. \& Showman, A. P. 2005, ApJ, 629, L45

Cooper, C. S. \& Showman, A. P. 2006, ApJ, 649, 1048

Cowan, N. B. \& Agol, E. 2008, ArXiv e-prints

Cowan, N. B., Agol, E., \& Charbonneau, D. 2007, MNRAS, 379, 641

Crossfield, I. J. M., Hansen, B. M. S., Harrington, J., et al., 2010, ApJ, 723, 1436

Deming, D., Seager, S., Richardson, L. J., \& Harrington, J. 2005, Nature, 434, 740

Desert, J.-M., Lecavelier des Etangs, A., H ebrard, G., et al., 2009, ApJ, 699, 478

Dobbs-Dixon, I., Agol, E., \& Burrows, A. 2011, In Prep.

Dobbs-Dixon, I., Cumming, A., \& Lin, D. N. C. 2010, ApJ, 710, 1395

Dobbs-Dixon, I. \& Lin, D. N. C. 2008, ApJ, 673, 513

Fortney, J. J., Shabram, M., Showman, A. P., et al., 2010, ApJ, 709, 1396

Harrington, J., Hansen, B. M., Luszcz, S. H., et al., 2006, Science, 314, 623

Knutson, H. A., et al., 2008, ApJ, 673, 526

Knutson, H. A., Charbonneau, D., Allen, L. E., et al., 2007, Nature, 447, 183

Knutson, H. A., Charbonneau, D., Cowan, N. B., et al., 2009, ApJ, 690, 822

Langton, J. \& Laughlin, G. 2007, ApJ, 657, L113

Langton, J. \& Laughlin, G. 2008, ApJ, 674, 1106

Mandel, K. \& Agol, E. 2002, ApJ, 580, L171

Menou, K. \& Rauscher, E. 2009, ApJ, 700, 887

Rauscher, E. \& Menou, K. 2010, ApJ, 714, 1334

Rauscher, E., Menou, K., Cho, J. Y.-K., Seager, S., \& Hansen, B. M. S. 2008, ApJ, 681, 1646

Seager, S. \& Sasselov, D. D. 2000, ApJ, 537, 916

Sharp, C. M. \& Burrows, A. 2007, ApJS, 168, 140

Showman, A. P., Cooper, C. S., Fortney, J. J., \& Marley, M. S. 2008, ApJ, 682, 559

Showman, A. P., Fortney, J. J., Lian, Y., et al., 2009, ApJ, 699, 564

Showman, A. P. \& Guillot, T. 2002, AA, 385, 166

Snellen, I. A. G., de Kok, R. J., de Mooij, E. J. W., \& Albrecht, S. 2010, Nature, 465, 1049

\section{Discussion}

E. Devinney: About the non-axis symmetric flows, are there for tidally-locked planets?

I. DobBs-Dixon: Yes, all the models I have been discussing have rotating rates that are tidally-locked to their orbital periods.

E. Guinan: GJ 581 has two super-Earth-type planets in or near the dM star's HZ. Have you carried out modeling of them? The circulation of the atmosphere from the hot (substellar) side to the cooler dark side would be important to habitability.

I. DobBs-Dixon: No, I have not pushed my models down to Earth-sized planets. There are a number of groups that are doing this, and indeed dynamics will play an important role in determining habitability. 